\title{
Development of Printed Sensors for Shoe Sensing Applications
}

\author{
Anindya Nag, Shilun Feng, Subhas Mukhopadhyay, Fellow, IEEE and Jürgen Kosel
}

\begin{abstract}
The paper presents the design and implementation of a low-cost shoe sensing system using laser-induced graphene sensors. Commercial polymer films were laser-induced to photothermally form graphene, which was then used as electrodes on Kapton tapes to form sensor patches. Experiments were then conducted with these sensor prototypes to validate its functionality as pressure sensors to be used in shoe sensing system. Different weights were tested with the developed system to ensure the capability of these sensor patches to be used as pressure sensing. The results look promising to be a system for monitoring the movement of a person wearing a shoe containing these low-cost pressure sensors.
\end{abstract}

Keywords-Graphene; Sensor; Laser writing; Pressure; Shoe sensing system;

\section{INTRODUCTION}

The use of sensors to improve the quality of life has been one of the primary goals for researchers in the current world. After the introduction of sensors into the application world [1], one of the goals was to make the use of the sensors to improve the quality of life. This needed continuous improvement of the performance of the sensors based on their size, linear range, sensitivity, power consumption, etc. Today, researchers all over the world are trying to develop different kinds of sensors having optimized performance. One of the primary constraints on the fabrication of sensors is the cost of production. It is generally noted that even though the cost of each sensor commercially available in the market is low, the equipment required to fabricate the sensors is very low. This includes the required number of steps, cost of the raw materials, cost associated with the equipment processing the raw materials, etc. These constraints increase the overall cost of each sensor. The work on reducing the cost constraint is being dealt by the researchers all over the world by addressing the techniques and raw materials for production. This paper presents one of the techniques to generate highly efficient low-cost capacitive sensors.

The structure of the sensors has been changing with time. Due to the popularity of sensors with semi-conductive substrates, silicon sensors have been used to a large extent. Even though these sensors served a lot of applications, there were some disadvantages like the high raw material cost, high power consumption and brittle nature of the sensors associated with the sensors.

Anindya Nag, Shilun Feng, and Prof. Subhas Mukhopadhyay are with Faculty of Science and Engineering, Macquarie University, Sydney, Australia. Prof. Jürgen Kosel is with Computer Electrical and Mathematical Sciences and Engineering Division, King Abdullah University of Science and Technology, Saudi Arabia.

(Email: anindya1991@gmail.com)
For these reasons, alternating materials have been processed with to develop sensors better than the existing ones. Sensors with flexible materials are one popular choice [2] that is being developed nowadays for its distinct advantages over the rigid ones. Lower cost, smaller size, better electrical and mechanical properties, higher efficiency in terms of sensitivity are some of the attributes that the sensors with flexible substrates have an advantage over the rigid ones. Even though there are many materials that are used to develop the substrates of the flexible sensors, some of the common ones are the polymer materials like PDMS [3], PET [4], PI [5], PMMA [6], etc. The processing of these materials has been done in different ways to develop sensor prototypes for specific applications. For these substrate materials, different conductive materials like carbon [7], silver [8], gold [9], aluminum [10], etc. have been used to develop the electrodes of the sensor. Similarly, different processing techniques like photolithography [11], screen printing [12], laser cutting [13] have been utilized to develop the sensors. The use of a particular technique depends on the resolution of the electrode and substrate thickness of the final sensor prototype. This paper describes the use of laser writing technique on polymer films to develop the conductive material for the electrodes [14]. The use of laser processing method to develop flexible sensors has been advantageous over other processes in many ways like less sample preparation time, the absence of any clean room requirements, smooth cuts, and non-requirement of any post-processing steps. This paper provides a two-step process to develop graphene sensors by using the laser writing technique to photo-thermally convert a polymer film to develop graphene, which was then used as electrodes on a sensor patch for experimental purposes. The use of graphene has increased exponentially in the last decade or so [15] after its recognition to have excellent electrical, mechanical and thermal properties. Graphene, is an allotrope of carbon [16], has been largely used as electrodes in a sensor. Few of the major applications of graphene over the years have been its uses in micro-capacitors [17, 18], strain sensors [19, 20] and electrochemical sensors [21, 22]. The electrode of the sensor shown in this paper is an extension of strain sensor where an external pressure is applied by the sciatic area of the feet over the sensing area of the patch to analyze the functionality of the graphene sensors to be used in a shoe sensing system. The sensors were attached to the sole of a shoe along with the placement of the attached conditioning circuit placed on the top of the shoelaces. There has been extensive work done on the development of shoe sensing systems [23-25] in recent years. Scientists all over the world have tried different ways of developing shoe sensors for tackling different problems related to walking posture [26, 27], and gait analysis [23, 28- 
30]. Among these vast topics covered by shoe sensors, one of the popular choices has been the use of pressure sensors in shoe sensing systems [31]. Pressure sensors have been placed on different regions $[32,33]$ of a shoe to determine the force exerted on that particular area while walking, running, etc. The use of shoe sensors has extensively covered different aspects till now. But there are certain disadvantages associated with these mentioned systems. Starting from the high fabrication cost of the systems, the developed systems are either very complex or require specialized condition and people to operate it. A simplified system is yet to be developed to date. The simplification of the system lies in the low cost of the processed raw materials to the simplified signal conditioning circuit to process the output of the sensor. The work described in this paper showcases the utilization of low-cost graphene sensor prototypes as pressure sensor patches to determine the walking of a person by analyzing the pressure exerted on the sensing area of the patch. This change in pressure while walking would be processed by a signal conditioning circuit and sent to the monitoring unit for further analysis.

\section{FABRICATION OF THE SENSOR PATCHES}

The fabrication process was carried out in the laboratory environment at constant temperature $\left(25^{\circ} \mathrm{C}\right)$ and humidity $(\mathrm{RH}$ $50 \%$ ) conditions. Low-cost commercial polymer films (Zibo Zhongnan Plastics Co., Ltd.)) were considered as the raw material for developing the electrode material. Figure 1 shows the schematic diagram of the overall fabrication process. The laser writing is done on the PI films generated graphene according to the assigned design of the laser system. This conductive material was transferred to sticky tapes for using it as electrodes on a sensor patch. Figures 2(1) -2(4) shows individual steps of the fabrication process. The polymer film was attached to a glass substrate with biocompatible tapes $(3 \mathrm{M}$ 810D Ruban Magique ${ }^{\mathrm{MC}}$ ) before placing it on the laser platform to restrict its movement during the laser induction process. Universal Laser Systems [34] was used to perform the laser induction process. Proper measurements were taken to exhaust the residual gases generated during the process. The optimization of the designed patterns was done by varying the given laser parameters. The designing of the electrodes was done on Coral DRAW, software that was integrated with the laser system. Three of the laser parameters, namely power $(\mathrm{W})$, speed $(\mathrm{m} / \mathrm{min})$ and $\mathrm{z}$-axis $(\mathrm{mm})$ were varied to optimize the design of the electrodes.

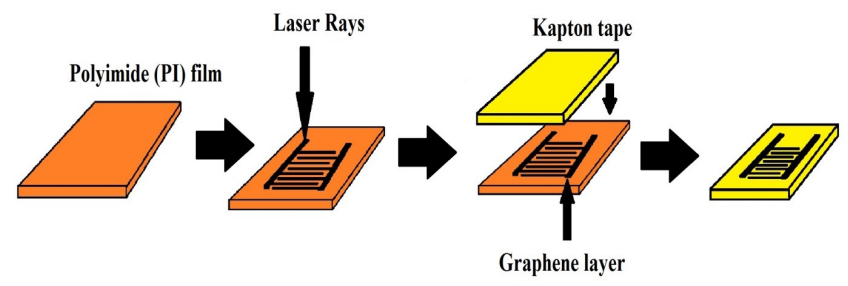

Figure 1: Schematic diagram of the fabrication steps of laser-induced graphene sensors.

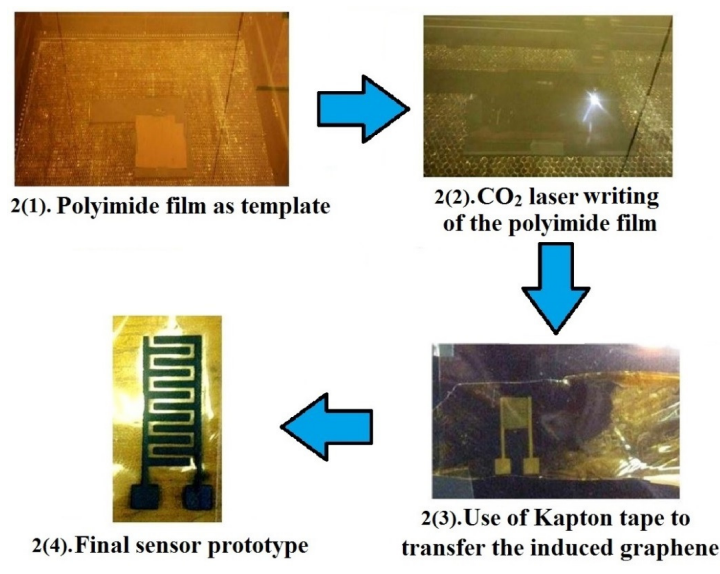

Figure 2: Individual steps for the fabrication of graphene sensor.

The power is defined as the amount of the energy of the laser exerted from the nozzle. Speed can be defined as the rate of movement of laser nozzle over the sample substrate. Z-axis defined the distance of the laser nozzle with the platform to adjust the focal point of the laser beam. The optimized parameters for this process were $9 \mathrm{~W}, 70 \mathrm{~m} / \mathrm{min}, 1 \mathrm{~mm}$. The laser induction process caused the breakage of the $\mathrm{sp}^{3}$ hybridized $\mathrm{C}-\mathrm{C}$ bonds of the PI film to form $\mathrm{sp}^{2}$ hybridized $\mathrm{C}$ $\mathrm{C}$ bonds in graphene. It is because graphene was generated in a power form, it would be really difficult to use it as electrodes in any sensor unless any stickiness was provided to hold the graphene. So, Kapton tapes were used for this purpose to transfer this graphene powder and use it as electrodes. Even though the commercial polymer films and Kapton tapes are of the same material (PI), there were two specific reasons for choosing the former material over the later one to develop the patches. The stickiness of the Kapton tapes would have tampered with the design and conductivity of the electrodes. The transfer of the graphene powder was done very carefully to preserve the design the electrodes. The Kapton tapes were placed over the graphene powder and manually pressed, starting from the sensing area of the sensor to the bonding pads. The conductivity and short-circuit tests were done before and after the transfer. The conductivity of the induced graphene was very high $\left(\sim 10^{\wedge} 4 \mathrm{~S} / \mathrm{m}\right)$. The difference in conductivities between the induced and transferred graphene was less than $20 \mathrm{mS} / \mathrm{m}$. The Kapton tapes with the transferred graphene were used as sensor patches for experimental purposes. The SEM images of the transferred graphene of one of the electrode fingers of its side and tip are shown in Figures 3 and 4 respectively. It is seen from the images that the electrode fingers of the transferred graphene came off smooth on the Kapton tapes. The edges of the electrodes were also perpendicular to the surface for which there was no requirement of any post-processing steps. The advantages of these developed patches are the high conductivity of the electrodes and the flexibility of the sensor patches. The electrical conductivity and bending diameter of the patches are $\sim 10^{\wedge} 4 \mathrm{~S} / \mathrm{m}$, and $6 \mathrm{~mm}$ respectively. Figure 5 shows the front and rear view of the final sensor patch. Six pairs of interdigitated fingers were present on the sensing area with an 
interdigital gap of 200 microns. The sensing surface area was $96 \mathrm{~mm}^{2}$ with a length of 500 microns and a width of 100 microns respectively.

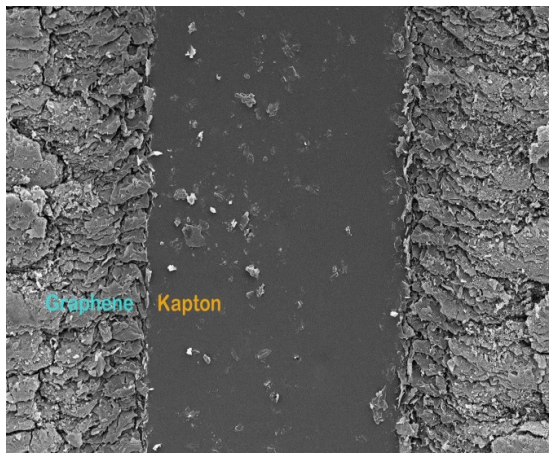

Figure 3: SEM image of top-view of the transferred graphene on Kapton tapes depicting the edges of the electrode fingers.

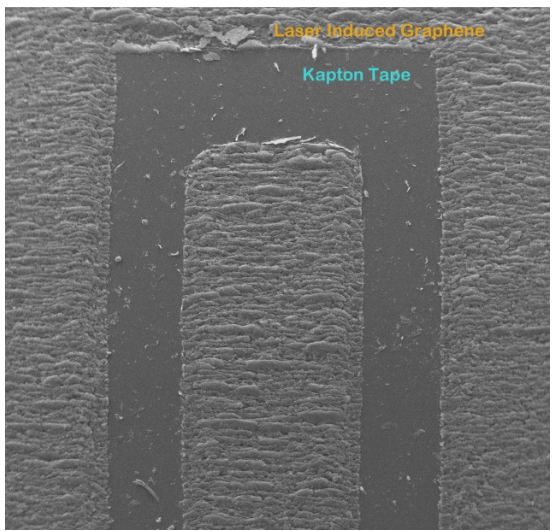

Figure 4: SEM image of top-view of the transferred graphene on Kapton tapes depicting one of the electrodes of the sensor.

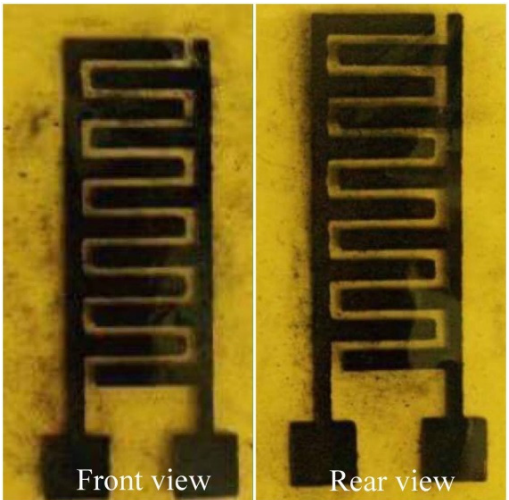

Figure 5: Front and rear view of the final fabricated sensor patch.

\section{WORKING PRINCIPLE OF THE ELECTRODES}

The electrodes of the developed sensor patches worked on the principle of deformation experienced by flexible sensors. Figure 6 shows the normal and expanded form of the sensor patch of which the latter is caused when a pressure is exerted on the patch. The deformation of the patch causes a change in the resultant current flowing through the sensor when an electric field is applied to it.

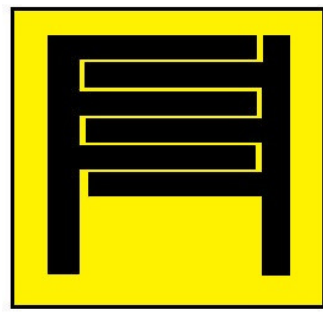

Normal State

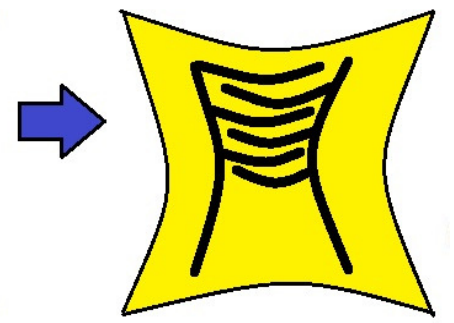

Deformed State
Figure 6: Schematic diagram depicting the change in structure from to normal to deformed state.

This change in current can be analyzed from schematic diagram shown in figure 7 [35]. It is seen from the figure that the $\mathrm{R}_{\text {sense }}$ and $\mathrm{C}_{\text {sense }}$ are the real $(\mathrm{R})$ and imaginary $(\mathrm{X})$ part of the impedance respectively. When any pressure is applied to the sensor patch, the resultant area (A) and interdigital distance $(d)$ changes, thus changing the reactive response of the sensor. This change can be determined by the above equations:

$$
\begin{gathered}
V_{\text {sense }}=I_{\text {in }} * R_{\text {series }} \\
Z_{\text {eq }}=V_{\text {in }} / I_{\text {in }}=\left(V_{\text {in }} / V_{\text {sense }}\right) * R_{\text {series }} \\
X_{\text {sense }}=Z * \sin \emptyset \\
R_{\text {sense }}=Z * \cos \emptyset-R_{\text {series }} \\
\text { And, } X_{\text {sense }}=w * C_{\text {sense }} \\
\text { where, } \mathrm{C}_{\text {sense }}=\frac{\varepsilon_{0}{ }^{*} \varepsilon_{\mathrm{r}} * \mathrm{~A}}{\mathrm{~d}}
\end{gathered}
$$

where, $\mathrm{V}_{\text {sense }}$ is defined as the voltage across the series resistance.

$\mathrm{Z}_{\mathrm{eq}}$ is the equivalent impedance.

Vin is the input voltage of the circuit.

$\Phi$ is the phase angle between the input voltage and input current.

$\mathrm{C}_{\text {sense }}$ in the output capacitance in Farads, F.

$\varepsilon_{0}$ is the absolute permittivity $\left(\varepsilon_{0}=8.854 * 10^{-12} \mathrm{~F} / \mathrm{m}\right)$.

$\varepsilon_{\mathrm{r}}$ is the relative permittivity.

A is the effective area.

$d$ is the effective interdigital distance between two consecutive electrodes.

The change in capacitance can be defined as the change caused due to the resultant area and interdigital distance. 


$$
\begin{gathered}
\Delta C_{\text {sense }}=f(\Delta A, \Delta d) \\
\Delta X_{\text {sense }}=w * \Delta C_{\text {sense }}
\end{gathered}
$$

where, $\Delta \mathrm{A}$ and $\Delta \mathrm{d}$ are the resultant areas and interdigital distance caused due to the deformation.

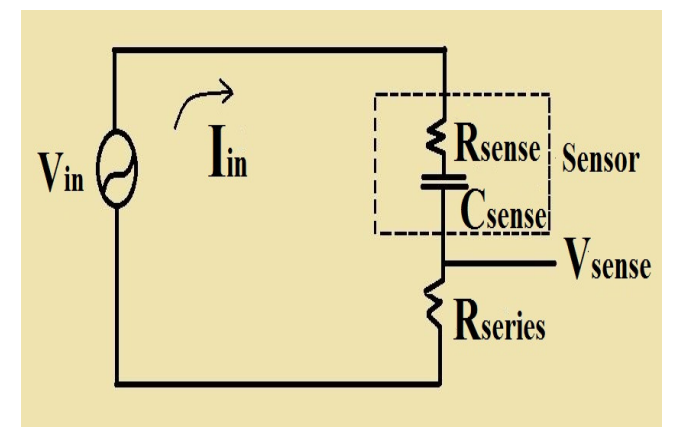

Figure 7: Schematic diagram of the electrical circuitry showing the change in current due to the change in output (sensed) impedance [35].

\section{EXPERIMENTAL RESULTS}

Initially, the profiling of the sensor patch was done to determine the linearity of the sensor patches. This was done by testing the sensor patches with small weights to analyze the changes happening due to the deformation of the sensor patch in terms of resistance and voltage. This was followed by testing the sensor patches with an LCR meter.

\section{a. Profiling of the sensor}

The sensor patch was initially tested at different weights to determine its response in terms of voltage and resistance values. Figures 8 and 9 depict the response of the sensor towards different weights ranging from $1 \mathrm{~kg}$ to $10 \mathrm{~kg}$.

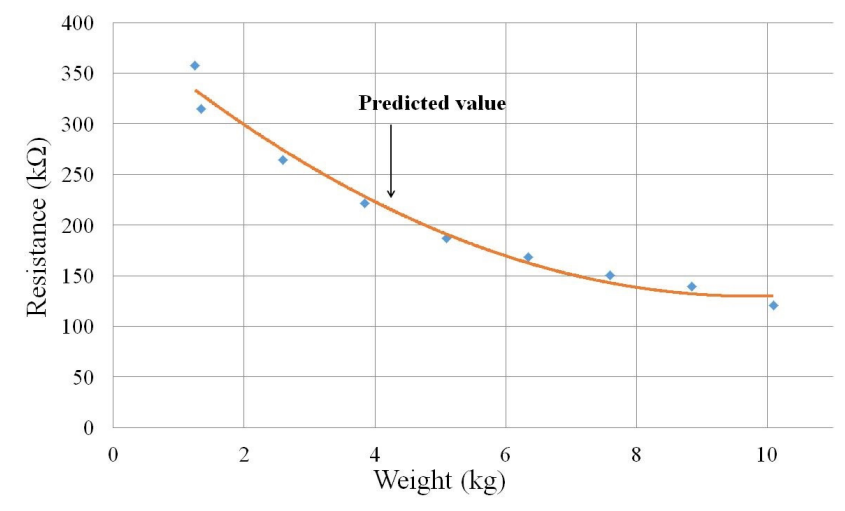

Figure 8: Response of the sensor patch to different weights in terms of resistance.

Even though it is known that the average weight of a person lies between $50 \mathrm{~kg}-70 \mathrm{~kg}$, which states that the weight of a single foot would be approximately $\pm 25 \mathrm{~kg}$, the limitation of the maximum weight to test the sensor was $10 \mathrm{kgs}$ was because of two reasons. Firstly, when a person walks, the weight of the whole leg $(25 \mathrm{~kg})$ is not on the sensor patch. The weight of the leg is distributed over the whole feet. And secondly, the idea to test the sensor with the weights is to validate the functionality towards pressure measurement. It is seen from figures 8 and 9 that the sensor is capable of responding in almost a linear way to different weights exerted on the sensing area of the patch. It is also seen from these two figures that the predicted value for the change in resistance and voltage readings of the sensor patches were very close to the actual values.

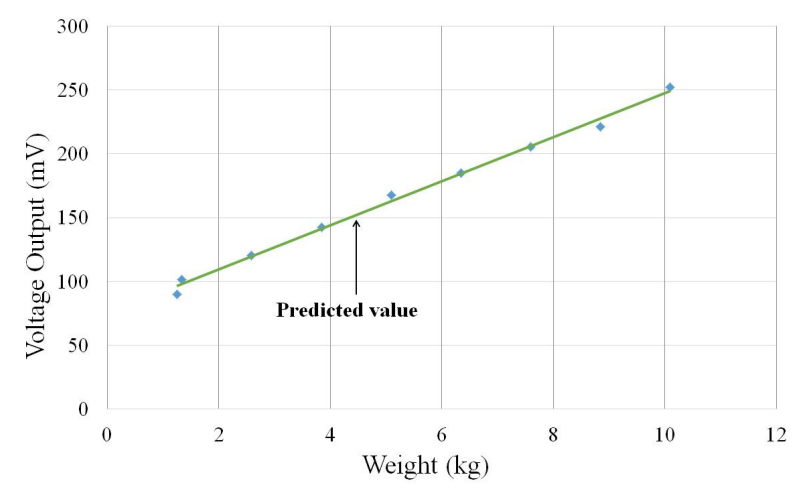

Figure 9: Response of the sensor patch to different weights in terms of voltage.

\section{b. Experimental results with LCR meter}

The sensor patch was tested with an LCR meter to validate its functionality for using it as a shoe sensing system. Due to the high conductivity of the electrodes, the sensor was capable of recognizing even pressures of small magnitude exerted on the sensing area of the patch. Figure 10 shows the connection of the LCR meter to the sensor. The change in impedance was analyzed by a HIOKI IM3536 High Precision Tester connected with Kelvin probes which in turn was connected to the bonding pads of the sensor patch. Due to the high flexibility of the patches, it was difficult to connect the Kelvin probes to the bonding pads. So, single-stranded flexible wires were connected to the bonding pads by a conductive carbon paint (BARE conductive ${ }^{\circledR}$ ELECTRIC PAINT), which in turn was connected to the Kelvin probes. A voltage of 1 Vrms was provided as an input to the sensor patch. The High Precision Tester was connected to a laptop via a USB cable to monitor the changes occurring with the exertion of pressure on the sensors in an alternative way. The data was collected in Microsoft Excel via automatic data acquisition algorithm. The experimental set-up is shown in figure 10. This figure shows the connection of the sensor patches to the High Precision Tester to the sensor patch. This was initially done to validate the functionality of the sensor patches to be used as pressure sensors in the shoe sensing system. Electrochemical Impedance Spectroscopy (EIS) was the algorithm associated with the High Precision Tester. EIS is a standard technique [36] used to characterize a material based on specific parameters. Among the different EIS technique available [37], 
frequency response analyzer (FRA) [38] is one of the popular ways to characterize the material over the range of frequencies. This technique has been used for the characterization of different materials including human physiological parameters [39], food products [40], salinity testing [41] and tactile sensing [42]. The advantages of using this technique are its simple, non-invasive and single-sided testing [43]. Figures 11 and 12 show the responses of the sensor patches for the force exerted on the sensor patch in a slow and rapid motion respectively. Two situations, "a" and "b", allocated for the two situations depicted in the figures represents the relaxed condition or pressure free condition and forced condition or the presence of the feet on the sensing patch condition respectively. The frequency values were swept between $2 \mathrm{kHz}$ to $10 \mathrm{kHz}$ to analyze the change in the reactance values. The sensor patch did not respond well to the frequencies below $2 \mathrm{kHz}$. One of the reasons for this behavior could be the connection of the Kelvin probes of the LCR meter to the single-stranded wires attached to bonding pads of the sensor patch. The reason for the decrease in reactance occurring due to the forced state can be attributed to the change in the dimensions of the sensor patch causing a change in $\mathrm{C}_{\text {sense }}$ (equation (6)) which as a result changes $\mathrm{X}_{\text {sense }}$ (equation (5)) It is seen from the figures that the sensor patch is capable of recognizing the two situations very distinctively. Repetitive movements were done for both the situations to ensure the repeatability of the response of the sensor patches.

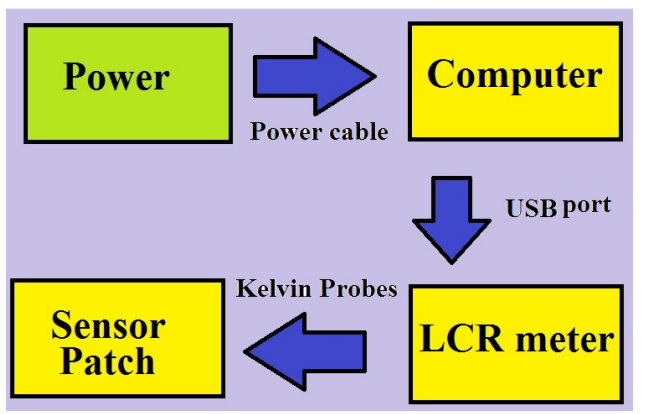

Figure 10: Schematic diagram depicting the process of data collection.

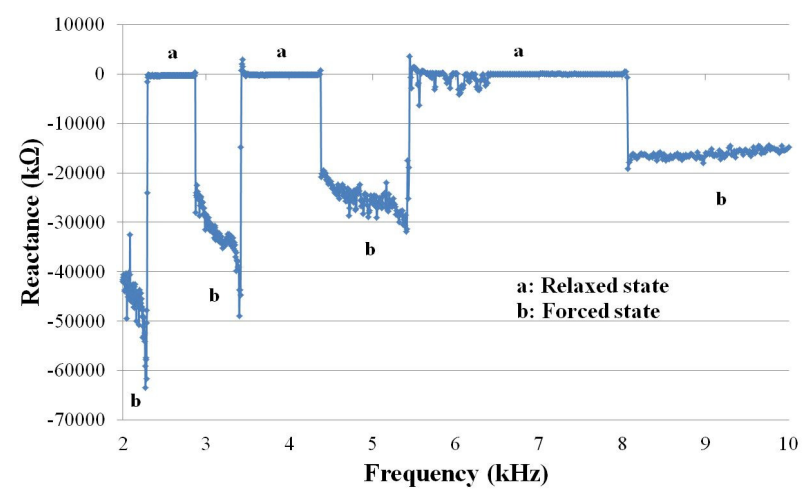

Figure 11: Response of the sensor patch in terms of reactance and frequency connected with the LCR meter with slow-motion expressed in the forced and relaxed state.

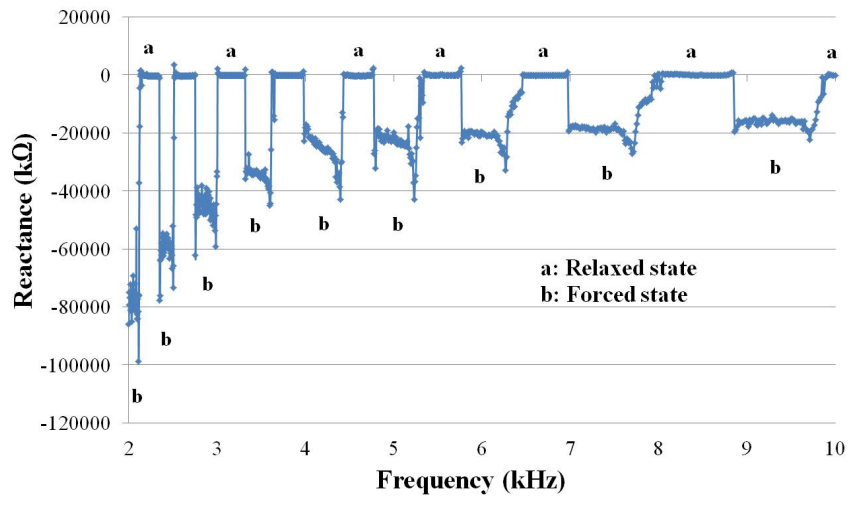

Figure 12: Response of the sensor patch in terms of reactance and frequency connected with the LCR meter with rapid motion expressed in the forced and relaxed state.is [7].

After testing the response of the sensor patches for repetitive cycles after in several experiments, they proved to be robust and sturdy in terms of wear and tear for this application. Also, the sensor patches had no memory effects in their responses after they were changed several times from one shoe to another while applying different amount of pressure.

\section{CONCLUSION}

This paper is an idealization of shoe sensing system with lowcost, laser-induced graphene sensors. The sensor patches were developed by using the electrodes formed by the photothermal conversion of the commercial polymer films. These sensor patches were then tested with the LCR meter to validate its functionality as pressure sensors which can be used for detection of movement for a shoe sensing system. However, there are some issues faced during the experimental procedures that should be rectified. Firstly, the range of sensor responses from the LCR meter in terms of reactance was not same during each cycle. This could be confusing for the monitoring unit once the reactance values for the forced state saturates to the values close to the relaxed state. Also, even though the sensor patches are very flexible in nature, there has to be testing done for their behavior and change in response with time. One of the ways is to implement a thresholding phenomenon for this purpose to clearly distinguish between the relaxed and the forced state. Secondly, even though the weight of different volunteers were tested with this system, all of them were perfectly normal having a proper walking posture. Thirdly, the use of LCR meter to validate this system should be replaced with an impedance analyzer embedded with a portable microcontroller based system to use it for realtime applications. The experiments with patients having an abnormal walking posture should be tried out to further validate this system. The rectification of these issues would be done and reported in near future work.

\section{ACKNOWLEDGMENT}

The authors would like to acknowledge King Abdullah University of Science and Technology (KAUST), Saudi Arabia to provide the research facilities to fabricate the sensor patches. 


\section{REFERENCES}

[1] S. M. Sze, Semiconductor sensors: Wiley New York, 1994.

[2] A. Nag, S. C. Mukhopadhyay, and J. Kosel, "Wearable Flexible Sensors: A Review," IEEE Sensors Journal, 2017.

[3] J.-H. Moon, D. H. Baek, Y. Y. Choi et al., "Wearable polyimidePDMS electrodes for intrabody communication," Journal of Micromechanics and Microengineering, vol. 20, no. 2, pp. 025032, 2010.

M.-x. Jing, C. Han, M. Li et al., "High performance of carbon nanotubes/silver nanowires-PET hybrid flexible transparent conductive films via facile pressing-transfer technique," Nanoscale research letters, vol. 9, no. 1, pp. 1-7, 2014.

[5] Y. Qin, Q. Peng, Y. Ding et al., "Lightweight, superelastic, and mechanically flexible graphene/polyimide nanocomposite foam for strain sensor application," ACS nano, vol. 9, no. 9, pp. 8933-8941, 2015.

[6] Y. Wang, R. Yang, Z. Shi et al., "Super-elastic graphene ripples for flexible strain sensors," ACS nano, vol. 5, no. 5, pp. 3645-3650, 2011.

[7] B. Natarajan, N. D. Orloff, R. Ashkar et al., "Multiscale metrologies for process optimization of carbon nanotube polymer composites," Carbon, vol. 108, pp. 381-393, 2016.

[8] S. Yao, and Y. Zhu, "Wearable multifunctional sensors using printed stretchable conductors made of silver nanowires," Nanoscale, vol. 6, no. 4, pp. 2345-2352, 2014.

[9] S. Gong, W. Schwalb, Y. Wang et al., "A wearable and highly sensitive pressure sensor with ultrathin gold nanowires," Nature communications, vol. 5, 2014

[10] W. Hu, S. N. Zhang, X. Niu et al., "An aluminum nanoparticleacrylate copolymer nanocomposite as a dielectric elastomer with a high dielectric constant," Journal of Materials Chemistry C, vol. 2 , no. 9, pp. 1658-1666, 2014.

[11] S. Khumpuang, H. Maekawa, and S. Hara, "Photolithography for minimal fab system," IEEJ Transactions on sensors and micromachines, vol. 133, no. 9, pp. 272-277, 2013.

[12] S. Khan, L. Lorenzelli, and R. S. Dahiya, "Bendable piezoresistive sensors by screen printing MWCNT/PDMS composites on flexible substrates." pp. 1-4.

[13] X. Li, and S. Garner, "Laser cutting of flexible glass." p. ATu3L. 1.

[14] J. Lin, Z. Peng, Y. Liu et al., "Laser-induced porous graphene films from commercial polymers," Nature communications, vol. 5, 2014.

[15] A. K. Geim, and K. S. Novoselov, "The rise of graphene," Nature Materials, vol. 6, no. 3, pp. 183-191, 2007.

16] W.-W. Liu, S.-P. Chai, A. R. Mohamed et al., "Synthesis and characterization of graphene and carbon nanotubes: A review on the past and recent developments," Journal of Industrial and Engineering Chemistry, vol. 20, no. 4, pp. 1171-1185, 2014.

[17] Z. Peng, R. Ye, J. A. Mann et al., "Flexible boron-doped laserinduced graphene microsupercapacitors," ACS nano, vol. 9, no. 6, pp. 5868-5875, 2015.

[18] H.-P. Cong, X.-C. Ren, P. Wang et al., "Flexible graphenepolyaniline composite paper for high-performance supercapacitor," Energy \& Environmental Science, vol. 6, no. 4, pp. 1185-1191, 2013

[19] Y. Wang, L. Wang, T. Yang et al., "Wearable and highly sensitive graphene strain sensors for human motion monitoring," Advanced Functional Materials, vol. 24, no. 29, pp. 4666-4670, 2014.

[20] S.-H. Bae, Y. Lee, B. K. Sharma et al., "Graphene-based transparent strain sensor," Carbon, vol. 51, pp. 236-242, 2013.

[21] A. T. Lawal, "Synthesis and utilisation of graphene for fabrication of electrochemical sensors," Talanta, vol. 131, pp. 424-443, 2015. S. Wu, Q. He, C. Tan et al., "Graphene-based electrochemical sensors," Small, vol. 9, no. 8, pp. 1160-1172, 2013.

[23] S. J. M. Bamberg, A. Y. Benbasat, D. M. Scarborough et al., "Gait analysis using a shoe-integrated wireless sensor system," IEEE Transactions on Information Technology in Biomedicine, vol. 12, no. 4, pp. 413-423, 2008.
E. S. Sazonov, G. Fulk, J. Hill et al., "Monitoring of posture allocations and activities by a shoe-based wearable sensor," Biomedical Engineering, IEEE Transactions on, vol. 58, no. 4, pp. 983-990, 2011.

C. E. Judy, L. D. Galuppo, J. R. Snyder et al., "Evaluation of an inshoe pressure measurement system in horses," American journal of veterinary research, vol. 62, no. 1, pp. 23-28, 2001.

H. Jonely, J.-M. Brismée, P. S. Sizer et al., "Relationships between clinical measures of static foot posture and plantar pressure during static standing and walking," Clinical Biomechanics, vol. 26, no. 8, pp. 873-879, 2011.

S. Yang, and Q. Li, "Inertial sensor-based methods in walking speed estimation: A systematic review," Sensors, vol. 12, no. 5, pp. 6102-6116, 2012.

A. Y. Benbasat, S. J. Morris, and J. A. Paradiso, "A wireless modular sensor architecture and its application in on-shoe gait analysis." pp. 1086-1091.

M. Gabel, R. Gilad-Bachrach, E. Renshaw et al., "Full body gait analysis with Kinect." pp. 1964-1967.

B. Mariani, M. C. Jimenez, F. J. Vingerhoets et al., "On-shoe wearable sensors for gait and turning assessment of patients with Parkinson's disease," Biomedical Engineering, IEEE Transactions on, vol. 60, no. 1, pp. 155-158, 2013.

K. F. Lei, K.-F. Lee, and M.-Y. Lee, "Development of a flexible PDMS capacitive pressure sensor for plantar pressure measurement," Microelectronic Engineering, vol. 99, pp. 1-5, 2012.

"Shoe Insole Sensor measuring pressure," https://www.2mel.nl/technology-incubation/shoe-insole-sensorpressure-shear-force-9dof/. Last accessed on 01.02.2018.

S. Sungkarat, B. E. Fisher, and A. Kovindha, "Efficacy of an insole shoe wedge and augmented pressure sensor for gait training in individuals with stroke: a randomized controlled trial," Clinical rehabilitation, vol. 25, no. 4, pp. 360-369, 2011.

"Universal Laser Systems," https://www.ulsinc.com/resources/laser-safety. Last accessed on 01.02 .2018 .

A. Nag, A. I. Zia, X. Li et al., "Novel Sensing Approach for LPG Leakage Detection: Part I-Operating Mechanism and Preliminary Results," IEEE Sensors Journal, vol. 16, no. 4, pp. 996-1003, 2016.

M. E. Orazem, and B. Tribollet, Electrochemical impedance spectroscopy: John Wiley \& Sons, 2011.

J. R. Macdonald, and E. Barsoukov, "Impedance spectroscopy: theory, experiment, and applications," History, vol. 1, no. 8, 2005. M. Ordonez, M. O. Sonnaillon, J. E. Quaicoe et al., "An embedded frequency response analyzer for fuel cell monitoring and characterization," IEEE Transactions on Industrial Electronics, vol. 57, no. 6, pp. 1925-1934, 2010.

A. Nag, S. C. Mukhopadhyay, and J. Kosel, "Flexible carbon nanotube nanocomposite sensor for multiple physiological parameter monitoring," Sensors and Actuators A: Physical, vol. 251, pp. 148-155, 2016

S. C. Mukhopadhyay, and C. P. Gooneratne, "A novel planar-type biosensor for noninvasive meat inspection," Sensors Journal, IEEE, vol. 7, no. 9, pp. 1340-1346, 2007.

A. Nag, S. C. Mukhopadhyay, and J. Kosel, "Sensing System for Salinity Testing Using Laser-induced Graphene Sensors," Sensors and Actuators A: Physical, 2017.

A. Nag, S. C. Mukhopadhyay, and J. Kosel, "Tactile Sensing From Laser-Ablated Metallized PET Films," IEEE Sensors Journal, vol. 17, no. 1, pp. 7-13, 2016.

E. Azzarello, E. Masi, and S. Mancuso, "Electrochemical impedance spectroscopy," Plant Electrophysiology, pp. 205-223: Springer, 2012. 